\title{
Critérios para a seleção de áreas prioritárias para a conservação em bacias hidrográficas
}

As delimitações metodológicas para seleção de áreas prioritárias devem ser bem estabelecidas, pois constituem importantes ações estratégicas de gestão. A seleção e mapeamento dessas áreas, ocorrem sobretudo a partir de um conjunto de critérios previamente determinado, segundo o alvo da conservação, subsidiando assim a tomada de decisão. Com o presente trabalho, objetivou-se analisar os principais critérios adotados para seleção de áreas prioritárias para conservação em bacias hidrográficas. Em termos metodológicos, foi realizada uma revisão de literatura, utilizando o banco de dados do Portal de Periódicos CAPES/MEC, Google Scholar e JTSOR. Os resultados demonstram um baixo número de artigos científicos publicados nos últimos dez anos abrangendo áreas prioritárias em bacias hidrográficas, evidenciando uma lacuna no conhecimento acerca de critérios objetivos para seleção dessas áreas; foram identificados e discutidos sete critérios macros: áreas de captação para abastecimento público, proximidade à rede de drenagem, possibilidade de conexão de fragmentos florestais, áreas com suscetibilidade a erosão, distância da malha viária, índice de urbanização e proteção de áreas ecologicamente relevantes. Nas conclusões, são destacadas recomendações para a elegibilidade dos critérios que compõem o processo de seleção de áreas prioritárias para conservação e restauração em bacias hidrográficas. Embora o processo de seleção dessas áreas possa apresentar limitações nas análises, o emprego de critérios consolidados pode representar um avanço em pesquisas vindouras que buscam a identificação de áreas prioritárias em bacias hidrográficas, visando sobretudo manter a quantidade, qualidade e a permanência do suprimento de água doce, atuando de maneira conjunta com os propósitos de conservação da biodiversidade.

Palavras-chave: Áreas Prioritárias; Restauração Florestal; Recursos Hídricos.

\section{Criteria for the selection of priority areas for conservation in watersheds}

\begin{abstract}
The methodological boundaries for selecting priority areas must be well established, as they are important strategic management actions. The selection and mapping of these areas occurs mainly from a set of criteria previously determined, according to the conservation target, thus subsidizing decision making. This study aimed to analyze the main criteria adopted for the selection of priority areas for conservation in watersheds. In methodological terms, a literature review was carried out, using the CAPES / MEC, Google Scholar and JTSOR database. The results demonstrate a low number of scientific articles published in the last ten years covering priority areas in watersheds, evidencing a gap in knowledge about objective criteria for selecting these areas; seven macro criteria were identified and discussed: catchment areas for public supply, proximity to the drainage network, possibility of connecting forest fragments, areas susceptible to erosion, distance from the road network, urbanization index and protection of ecologically relevant areas. In the conclusions, recommendations are highlighted for the eligibility of the criteria that make up the process of selecting priority areas for conservation and restoration in watersheds. Although the selection process of these areas may present limitations in the analysis, the use of consolidated criteria may represent an advance in future research that seeks to identify priority areas in watersheds, aiming above all to maintain the quantity, quality and permanence of the water supply. sweet, acting in conjunction with the purposes of biodiversity conservation.
\end{abstract}

Keywords: Priority Areas; Forest Restoration; Water Resources.

Topic: Engenharia Ambiental

Reviewed anonymously in the process of blind peer.
Received: 04/03/2021

Approved: 24/03/2021

Julliana Oliveira de Araujo Pereira (iD

Instituto Federal Fluminense, Brasil

http://lattes.cnpq.br/2718358135044755

http://orcid.org/0000-0003-1062-766X

car.julliana@gmail.com

David de Andrade Costa (i)

Instituto Federal Fluminense, Brasil

http://lattes.cnpq.br/0403441577562653

http://orcid.org/0000-0003-1814-5892

david.costa@iff.edu.br

Luis Felipe Umbelino dos Santos

Instituto Federal Fluminense, Brasil

http://lattes.cnpq.br/0834418937830253

lfumbelino@gmail.com

\section{Referencing this:}

PEREIRA, J. O. A.; COSTA, D. A.; SANTOS, L. F. U.. Critérios para a seleção de áreas prioritárias para a conservação em bacias hidrográficas. Revista Ibero Americana de Ciências Ambientais, v.12, n.3, p.333-348, 2021. DOI: http://doi.org/10.6008/CBPC21796858.2021 .003 .0028 


\section{INTRODUÇÃO}

A proteção ao meio ambiente é um dos maiores desafios globais e uma ideia central nos Objetivos de Desenvolvimento Sustentável da ONU (WOOD et al., 2018). Em resposta às transformações antrópicas impostas aos sistemas naturais, inúmeras estratégias de restauração e conservação de ecossistemas tem sido desenvolvidas (SANTOS et al., 2020; COSTA et al., 2019). Em especial, as soluções baseadas na natureza têm sido recomendadas como uma forma de manutenção de serviços ecossistêmicos (NÉTO et al., 2020). Porém, o enfrentamento desse desafio global depende de variados recursos que, frequentemente escassos, precisam ser priorizados (LIQUETE et al., 2016; FRANTZESKAKI, 2019).

Para tanto, as metas e o objetivo de conservação precisam ser bem definidos através do estabelecimento de prioridades. A preservação de espécies, comunidades, processos ecológicos ou serviços ecossistêmicos, atuam como importantes indicadores ecológicos capazes de representar o estado dos alvos de conservação, o que torna esta temática ampla e profunda dentro de vários aspectos (METZGER et al., 2006).

No final dos anos 80, Norman Myers foi o precursor de estudos que transformariam o cenário da conservação global, dedicando esforços para identificar as regiões mais importantes para proteção da biodiversidade, chamadas de hotspots. Dois critérios foram estabelecidos para definição dos hotspots: I) regiões que apresentam concentrações excepcionais de espécies com altos níveis de endemismo; e II) regiões que enfrentam ameaças de destruição de habitat (MYERS, 1990; MYERS et al., 1988). Contudo, Myers não estabeleceu critérios quantitativos para definição de um hotspot, tão pouco como eles seriam conservados em virtude de sua grande extensão (MYERS et al., 2000). O incremento das atividades humanas no interior de hotspots, assim como as perturbações hidrológicas representam uma das principais ameaças à biodiversidade (ROUX et al., 2019). Nesse sentido, ações conservacionistas e/ou de restauração foram empregadas tendo em vista diminuir a extinção de espécies e promover a proteção de áreas menores em vários locais importantes para conservação (MYERS, 1990; MYERS, 1988; REID, 1998).

No Brasil, as diretrizes para a identificação das Áreas Prioritárias para a Conservação (APC) foram instituídas pelo Decreto № 5.092 de 21/05/2004 e pela Portaria № 126 de 27 de maio de 2004 no âmbito das atribuições do Ministério do Meio Ambiente (MMA). Ambos seguem a abordagem do Planejamento Sistemático da Conservação (PSC), que propõe alcançar metas para conservação, seguindo princípios como insubstituibilidade, representatividade, complementariedade, flexibilidade, e vulnerabilidade de uma região, cujo o intuito é aumentar a representatividade das áreas protegidas, atendendo os alvos de conservação a partir dos atributos ecológicos da área (BRASIL, 2017; MARGULES et al., 2000).

A abordagem do PSC descrita por Margules et al. (2000), atua como um suporte à tomada de decisão e à negociação, combinando informações sobre os alvos de conservação, custos da conservação e aspectos da paisagem, retratando critérios fundamentais para preservação da diversidade biológica. As ferramentas utilizadas nesta priorização encontram-se em contínuo aperfeiçoamento, e podem variar de acordo com as avaliações para cada bioma. Destaca-se a importância da participação da sociedade e o uso de ferramentas 
tecnológicas, como os Sistemas de Informação Geográfica (SIGs), que fornecem subsídios à tomada de decisão e reduzem a subjetividades das ações (BRASIL, 2005; BRASIL, 2017).

As delimitações metodológicas para seleção de áreas prioritárias (APs) devem ser muito bem estabelecidas, pois constituem importantes ações estratégicas de gestão (MELLO et al., 2018). A seleção e mapeamento dessas áreas, ocorrem sobretudo a partir de um conjunto de critérios que devem ser determinados de acordo com o objetivo do processo, subsidiando a tomada de decisão (VETTORAZZI et al., 2016). Na prática, geralmente os critérios selecionados são muitas vezes vagos e simplificados, não retratando com fidelidade a área analisada (SIMONCIC et al., 2013).

As diferentes abordagens para seleção de APs visam sobretudo preservar os recursos naturais, porém com diferentes enfoques que vão desde a proteção de espécies em extinção, até propósitos turísticos como a preservação de paisagens de grande beleza cênica. Tais abordagens focam em temas específicos e, muitas vezes, carecem de uma perspectiva mais integradora, tais como os recortes espaciais das bacias hidrográficas. Estas unidades de paisagem são utilizadas para a gestão territorial e integram a água com os demais componentes ecossistêmicos de uma bacia. Nesse sentido, destaca-se o a função das florestas, que corroboram com a gestão de recursos hídricos, exercendo influência sobre a quantidade, qualidade e a permanência do suprimento de água doce, atuando de maneira conjunta com os propósitos de conservação da biodiversidade (BIDEGAIN, 2019).

Considerando a necessidade de adoção de critérios objetivos para definição de áreas prioritárias para conservação, foi realizada uma revisão de literatura com o objetivo de verificar os principais critérios utilizados para seleção dessas áreas em bacias hidrográficas. Os recursos hídricos são evidenciados como elementos de integração para os mais variados objetivos de conservação e restauração. Como resultados foram identificados e discutidos sete critérios macros: áreas de captação para abastecimento público, proximidade à rede de drenagem, possibilidade de conexão de fragmentos florestais, áreas com suscetibilidade a erosão, distância da malha viária, índice de urbanização e proteção de áreas ecologicamente relevantes .

\section{METODOLOGIA}

A pesquisa foi realizada utilizando a técnica bibliométrica, a qual ocorreu no mês de abril e maio/2019. Utilizamos a base de dados do Portal de Periódicos CAPES/MEC, onde empregamos o termo de busca: 'conservation priority areas', resultando em 64.827 artigos científicos no período de 2009 a 2019. Em seguida uma nova busca foi efetuada, com o termo 'conservation priority areas', no campo de busca 'no título', onde resultou em 198 artigo científicos. Realizamos a leitura exploratória do conteúdo dos artigos, a fim de embasar a criação de algumas categorias de análise. As categorias criadas tiveram por finalidade separar os artigos por temáticas ou grupos de conservação, e assim compreender a lacuna de estudos existentes.

Uma nova busca foi realizada devido à lacuna identificada em pesquisas na área temática de 'bacias hidrográficas'. Para o levantamento dos artigos científicos, foram utilizados os seguintes termos de busca: 
'conservation priority áreas', 'Water resource', conservation priority areas and Watershed, 'Watershed management', 'Land prioritization and Water resource', 'Ecological Restoration', 'Soil and water conservation', 'Multi criteria evaluation and Watershed', 'Geographic information systems', 'Forest restoration and Watershed'. Utilizamos as plataformas Periódicos CAPES/MEC, Google Scholar e JTSOR e selecionamos os artigos científicos do período de 2009 a 2019. Buscamos, ainda, os documentos temáticos acerca de projetos e programas governamentais brasileiros.

A partir dos artigos e documentos selecionados, realizamos uma nova pesquisa, que teve o intuito de sustentar a revisão bibliográfica referente à temática em questão. Buscamos levantar os principais critérios (fatores) para seleção de APs, e agrupamos em sete categorias generalistas os critérios mais similares entre si, com maior representatividade frente ao objetivo delimitado, considerando a diversidade de variáveis as quais são possíveis analisar em cada critério. Ressaltamos ainda que, todos os critérios selecionados visam o incremento de ações de conservação e/ou restauração florestal, tendo em vista o benefício direto aos recursos hídricos.

\section{RESULTADOS E DISCUSSÃO}

\section{Grupos temáticos para áreas prioritárias}

Os trabalhos científicos foram agrupados em quatorze temáticas, de acordo com o objetivo das pesquisas desenvolvidas nas áreas denominadas prioritárias para a conservação (Figura 1). As temáticas que mais se destacaram foram Herpetofauna e Avifauna, que representam $27 \%$ do total de artigos analisados. Estudos apontam que os animais que compõe estes grupos, se destacam devido à tolerância e sensibilidade térmica, atuando como bons indicadores quanto a qualidade do ambiente em ecossistemas terrestres (CAMACHO, 2012; BUCKLEY et al., 2012). O grupo dos animais ectotérmicos, realizam a termorregulação comportamental, tendo a temperatura corporal alterada de acordo com as condições climáticas, influenciando no desempenho fisiológico e determinando seu modo de vida. Alguns estudos retratam ainda que, a presença ou ausência desses animais são capazes de indicar cenários de degradação ambiental (BARBOSA et al., 2017).

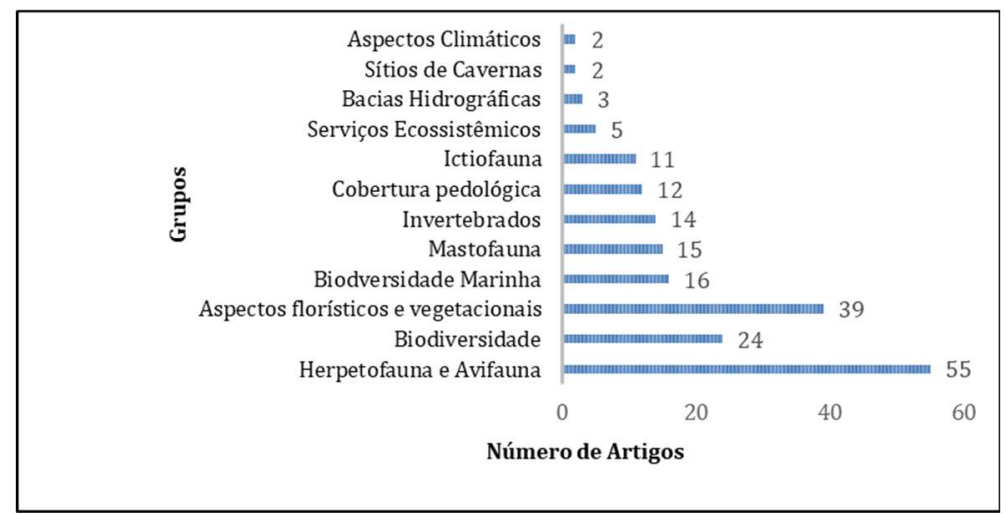

Figura 1: Grupo de temáticas versus número de artigos científicos publicados.

A segunda categoria que apresentou um grande volume de trabalhos, cerca de $20 \%$, apontam para os aspectos florísticos e vegetacionais no âmbito da conservação, em diferentes perspectivas. Pode-se 
perceber trabalhos que versam sobre representações de plantas em uma biorregião (WILLIAMS et al., 2009); mudanças na cobertura florestal devido a exploração madeireira, associadas ao estabelecimento de políticas florestais para expansão da cobertura florestal em uma APC (HOEK et al., 2014); modelagem macroecológica para identificar prioridades para conservação e manejo (RAPACCIUOLO, 2018); identificação de áreas de floresta tropical com probabilidade de conter a maior concentração espécies raras, espécies endêmicas e famílias de angiospermas primitivas (MOKANY et al., 2014), entre outros. Nesse sentido, pode-se inferir que grande parte dos estudos que envolvem os aspectos florísticos e vegetacionais visam obter dados em torno da perda de habitat em determinadas regiões, fornecendo informações importantes para a implementação de novas áreas protegidas, programas de restauração florestal e serviços ecossistêmicos.

Percebe-se também mediante aos dados apresentados (Figura 1), que apenas $6 \%$ dos trabalhos publicados retrataram a temática de cavernas, clima, bacias hidrográficas e serviços ecossistêmicos. Estes temas possivelmente são tratados com relevante interesse ecológico sem necessariamente serem incluídos na temática de AP.

Os resultados demonstraram que, os trabalhos científicos, em sua maioria foram direcionados a desenvolver a temática de APC com grupos taxonômicos e ecológicos, sobretudo do ponto de vista da biodiversidade. E um número menor de trabalhos foram conduzidos a trabalhar APC em bacias hidrográficas, sítios de caverna, aspectos climáticos e serviços ecossistêmicos. Contudo, esse baixo quantitativo de trabalhos na perspectiva de bacias hidrográficas, não conferem uma menor importância ao tratamento de recursos hídricos, pois muitos estudos ecológicos possuem como habitat os ecossistemas aquáticos. Nesse sentido, realizamos uma segunda análise, onde buscamos levantar os principais critérios (fatores) e as recomendações da literatura científica para seleção de locais prioritários para o incremento de ações de conservação e/ou restauração florestal, tendo em vista o benefício direto aos recursos hídricos.

\section{Priorização de áreas para manejo em bacias hidrográficas}

Devido às suas características naturais, a bacia hidrográfica possibilita o desenvolvimento de ações que visam a conservação dos recursos hídricos e terrestres. O gerenciamento de bacias hidrográficas, requer a compreensão acerca das relações entre: diferentes usos do solo; declive; geomorfologia e processos erosivos, o que a torna uma importante unidade espacial. Contudo, para um gerenciamento efetivo, a área da bacia é submetida a divisões em sub-bacias a partir da sua rede de drenagem (MESHRAM et al., 2017). É importante estabelecer estratégias para mitigar os crescentes impactos ambientais gerados ao longo do tempo nas bacias hidrográficas, assim como promover a sustentabilidade ambiental, social e econômica dos recursos hídricos. Contudo, a escassez de recursos financeiros torna-se um fator limitante que requer a determinação de prioridades para investimentos em ações conservacionistas (BEGER et al., 2015).

A literatura apresenta inúmeros trabalhos conduzidos no âmbito da agricultura agroflorestal, manejo florestal e reflorestamento, visando beneficiar os recursos hídricos como, Randhir et al. (2001), Bocher (2010), Ruiz (2015), Silva et al. (2017), Almeida et al. (2019), Mafra et al. (2020). No Brasil e na América Latina, alguns projetos de Pagamento por Serviço Ambiental hídrico (PSA) ganharam destaque, tendo em vista a 
conservação e restauração da cobertura vegetal como um critério para melhoria da qualidade e disponibilidade hídrica. O projeto implantado na cidade de New York desde 1997, foi o precursor em utilizar os serviços ecossistêmicos visando a provisão de água de uma grande metrópole; no Brasil, o pioneiro foi o Projeto "Conservador das Águas", que serviu de parâmetro para outras iniciativas dentro do país, sendo adequado às particularidades de cada localidade (MATSUOKA, 2019).

No contexto da restauração da paisagem, as iniciativas requerem a inclusão de diferentes critérios que contribuem nas decisões, e métodos que se integrem junto a esses critérios, com a finalidade de definir áreas que sejam prioritárias para desempenhar as ações de conservação na paisagem (VETTORAZZI et al., 2016). Convencionalmente, os critérios são divididos em fatores e restrições. Os fatores conduzem para o objetivo da restauração e representam as características críticas da paisagem que se tem a finalidade de alcançar. Enquanto as restrições, auxiliam colocando limites espaciais em AP, sinalizando os locais em que elas não deveriam ocorrer (SILVA et al., 2017).

A priorização de áreas representa uma alternativa eficaz para o manejo de bacias hidrográficas, sobretudo, a partir da espacialização das ações, que são associadas à medidas para conservação da água e solo, alternativas para abastecimento, infraestrutura hídrica, saneamento, dentre outras (IGAM, 2018; MESHRAM et al., 2019). A capacidade de integrar e analisar diferentes informações geográficas da paisagem em ambiente SIG, permite a construção de mapas de prioridade em um tempo curto e com grande grau de confiabilidade (VALENTE et al., 2017). Podemos citar os estudo de Silva et al. (2017) que buscaram identificar AP para conservação florestal em bacias hidrográficas visando manter a disponibilidade hídrica em quantidade e qualidade para o abastecimento público; Gashaw et al. (2018) que identificaram e priorizaram áreas passíveis para ações de conservação do solo em bacias hidrográficas na Etiópia através do estudo dos riscos de erosão e Meshram et al. (2017) que aplicaram a análise de parâmetros morfométricos, baseados na abordagem em Análises de Componentes Principais (ACP), visando a conservação do solo e da água para priorização de sub-bacias hidrográficas na Índia.

\section{Critérios para priorização de áreas}

Nesse sentindo, um levantamento desenvolvido por Gjorup et al. (2014), evidenciou que as etapas metodológicas envolvidas em programas de seleção de AP são geralmente divididas em dois grupos, o de elegibilidade - cujos critérios visam selecionar o público-alvo de acordo com o que é proposto pelo programa; e a priorização de áreas - onde propõe-se em identificar regiões prioritárias, que podem ser municípios, bacias hidrográficas, Áreas de Preservação Permanente (APPs) ou áreas definidas previamente pelo programa. Uma infinidade de outros critérios também são empregados para auxiliar na tomada de decisão, visando à seleção de áreas que apresentam prioritárias condições para o desenvolvimento de ações.

Assim, foram agrupados em sete categorias ambientais os critérios que estavam de acordo com a análise das principais características observadas nos artigos científicos para identificação das AP. Os critérios selecionados foram considerados significativos por serem indicadores de relevância das áreas; oferecerem contribuição ao regime das águas e capacidade para recuperação de bacias; quais sejam: 


\section{Seleção de bacias ou sub-bacias para abastecimento de água}

Um dos principais bens e serviços do ecossistema fornecidos por bacias hidrográficas é o abastecimento de água potável, pois eles são responsáveis por regular os fluxos hídricos e melhorar a qualidade da água, configurando um dos serviços mais concretos e valiosos (POSTEL et al., 2005; PAUDYAL et al., 2019). Bacias hidrográficas cujo uso prioritário é o abastecimento público de água, representa um importante critério a ser analisado no processo de seleção de AP. Ações implementadas em áreas rurais, refletem substancialmente no meio urbano para controle de enchentes e erosão do solo. Dispor de recursos para proteção dos mananciais, incluindo as águas interiores, subterrâneas ou superficiais, são estratégias positivas para garantir o abastecimento público para sociedade, uso industrial e hidroenergético (POSTEL et al., 2005; INEA, 2018).

Quando objetivamos em priorizar áreas em bacias hidrográficas que realizam serviços de abastecimento, estamos buscando, sobretudo, manter a qualidade e disponibilidade de água. Ao desenvolver um modelo de priorização de terras na micro bacia hidrográfica do Rio Ware, em Massachusetts, EUA, Randhir et al. (2001) buscou a proteção da qualidade da água para abastecimento. Esse modelo levou em consideração o tempo de circulação da água de runoff; a distribuição de um poluente pela bacia e quais locais se apresentam mais sensíveis à degradação. Para tanto, foi utilizado os níveis e pesos dos critérios para constituir o índice de priorização para cada localidade na bacia hidrográfica.

Os autores apresentam um método baseado em Sistema de informação geográfica (SIG), e na relação entre critérios e seus efeitos na qualidade da água. No que tange a tomada de decisão, foi utilizado a técnica da Análise Hierárquica Analítica $(\mathrm{AHA})$, onde os especialistas atribuem pesos usando uma escala de comparação que varia de 1 a 9. Segundo os autores, de acordo com o ajuste de critérios e pesos nessa abordagem, é possível realizar adaptações no processo da tomada de decisões sobre a proteção do uso do solo, como, a preservação de áreas florestais, a proteção de habitats críticos para a vida silvestre, criação de áreas recreacionais e planejamento ecológico-econômico (RANDHIR et al., 2001).

No estado do Rio de Janeiro, a Política Estadual de Recursos Hídricos, definida pela Lei no 3.239/1999 acrescenta, além dos instrumentos de gestão instituídos, o Programa Estadual de Conservação e Revitalização de Recursos Hídricos (PROHIDRO). O PROHIDRO tem por objetivo propiciar a revitalização e a conservação integral dos recursos hídricos, na perspectiva do ciclo hidrológico, tendo a bacia hidrográfica como unidade de planejamento e trabalho (RIO DE JANEIRO, 1999). Regulamentado pelo Decreto $\mathrm{n}$ o 42.029/2011, no âmbito do PROHIDRO, um mecanismo de Pagamento por Serviços Ambientais foi instituído, denominado Programa Estadual de Pagamento por Serviços ambientais (PRO-PSA) (RIO DE JANEIRO, 2011).

Logo, alguns programas e projetos bem sucedidos foram implementados em bacias hidrográficas no estado do RJ, visando preservar os recursos hídricos, sobretudo as áreas relevantes para o abastecimento público a partir da priorização de áreas. A título de exemplo, o projeto proposto pelo Comitê de Integração da Bacia Hidrográfica do Rio Paraíba do Sul - CEIVAP para PSA Hídrico, visa contribuir para melhoria da qualidade e quantidade de recursos hídricos em toda bacia; outra iniciativa já implantada é o Programa 
Produtor de Água e Floresta (PAF), um instrumento de PSA na Bacia Hidrográfica do Rio Guandu, onde busca incentivar práticas de conservação e restauração dos recursos naturais, sobretudo de mananciais estratégicos e florestas (PAGIOLA et al., 2013; RUIZ, 2015; INEA, 2018).

Em ambos os projetos, foi necessário o mapeamento e avaliação do uso do solo e cobertura vegetal da bacia, obtidos através de bases cartográficas, imagens de satélite, coleta de dados associados à caracterização da estrutura fundiária e agrícola, indicadores hidrológicos e bioindicadores. A integração destes dados ocorreu em ambiente SIG, contribuindo para a tomada de decisão no processo de avaliação e seleção das APs, resultando em ações de conservação e restauração florestal, além do pagamento ao Provedor do Serviço Ambiental (RUIZ, 2015). Quanto ao processo de hierarquização dos pesos e valores para cada critério, a relevância da bacia para o abastecimento é considerada o critério mais relevante.

Para tanto, a seleção da bacia ou micro bacia que em o projeto foi desenvolvido se deu a partir de três eixos centrais, a saber: relevância das áreas para produção e abastecimento de água; importância para a conservação da biodiversidade e identificação de parceiros institucionais nos territórios. Para cada eixo foi definido um conjunto de indicadores e seus respectivos pesos, que após serem somados possibilitou a equipe técnica definir a AP com maior facilidade e respaldo (PAGIOLA et al., 2013).

\section{Proximidade à rede hidrográfica}

A proximidade à rede hidrográfica representa um critério significativo à conservação dos recursos hídricos através da restauração florestal (VETTORAZZI et al., 2016). Uma vez que a cobertura vegetal contribui sobremaneira na produção de água numa bacia hidrográfica, assim como para o exercício de funções ecológicas essenciais (QIU et al., 2015). Nesse sentido, áreas mais próximas à corpos hídricos passam a ser relevantes para proposição de ações de recomposição e conservação florestal, considerando também os benefícios oferecidos à estrutura da paisagem, possibilidade de conexão de fragmentos e proteção hidrológica.

Ao analisar a qualidade da água sob a influência da paisagem nas bacias de captação e zona ripária, localizados em regiões agrícolas na bacia do Corumbataí no Brasil, Mori et al. (2015) observaram que, as florestas fragmentadas próximas à bacias de captação e zonas ribeirinhas não são capazes de manter a proteção dos ecossistemas aquáticos, resultando em alterações na qualidade da água. Investigando áreas próximas à rede hidrográfica tendo em vista a valorização de regiões para ações de planejamento ecológico e a conservação dos recursos hídricos, Comino et al. (2016) associou o SIG à avaliação multicritérios (AMC), obtendo resultados significativos no âmbito da ecologia da paisagem e na gestão do uso do solo, demonstrando a contribuição dos recursos hídricos para a conectividade da paisagem.

Para análise deste fator, são gerados mapas baseados em SIG a partir do plano de rede hidrográfica local, que contemplam sobretudo os cursos d'água e represas. A elaboração do plano de rede hidrográfica é realizada com o auxílio de cartas topográficas, acrescido da sua digitalização em tela e imagens de satélites. Em seguida são produzidos mapas de proximidade, que geralmente são relacionados com a proximidade à cobertura florestal (VALENTE et al., 2017). 


\section{Proximidade à regiões florestadas}

As florestas nativas desempenham diferentes funções na dinâmica dos processos hidrológicos, como o controle da erosão e aporte de sedimentos, regulação da quantidade e qualidade da água (PINHEIRO et al., 2019; TAMBOSI et al., 2015). A presença de matas ciliares contribui com a manutenção da biodiversidade terrestre e funcionam como corredores ou áreas de hábitat para diversas espécies de animais e plantas (PARDINI et al., 2010; CASSIANO et al., 2013). Todavia, as mudanças no uso do solo, tem produzido impactos diretos à biodiversidade, resultando na fragmentação dos ecossistemas naturais.

O estado de conservação dos fragmentos florestais fornece informações importantes para subsidiar o planejamento de programas de reflorestamento, com vista a aumentar a conectividade da paisagem através de corredores ecológicos (LIU et al., 2019; MITCHELL et al., 2015). Áreas próximas à fragmentos florestais são interessantes, uma vez que promovem a conexão destes e representam ganhos para biodiversidade regional, bem como o desenvolvimento a longo prazo dos processos ecológicos (CALEGARI et al., 2010). A proximidade à fragmentos de vegetação foi um dos fatores adotados em estudos que utilizaram a AMC em associação com a Combinação Linear Ponderada (WLC) por Valente et al. (2017).

A inserção deste fator em estudos que visam a construção de mapas para priorização de áreas, são baseados em SIG. A identificação de áreas próximas à fragmentos florestais ou a distância entre fragmentos é viabilizada a partir de mapas de uso e cobertura do solo. Bochner (2010) utilizou o software ArcGIS 9.3 e a ferramenta Distance/Euclidean Distance para calcular a distância entre os fragmentos florestais. De modo geral, as áreas mais próximas à fragmentos e à rede de drenagem recebem o indicativo de maior prioridade (VALENTE et al., 2017), e a distância entre os fragmentos apresenta relevância no cálculo de prioridade (BOCHNER, 2010).

\section{Suscetibilidade a erosão}

A suscetibilidade à erosão está diretamente relacionada à erodibilidade, devido às propriedades do solo. Esse critério é fundamental no processo de identificação de APs à restauração florestal ${ }_{L}$ visando à conservação de recursos hídricos. Áreas que sinalizam alta suscetibilidade à erosão, apresentam uma grande quantidade de sedimentos que podem acometer os mananciais, cursos d'água e reservatórios. É comum que essas áreas sejam classificadas como, áreas de alta prioridade (VETTORAZZI et al., 2016).

Ao caracterizar a sub-bacia hidrográfica mais susceptível a erosão do solo no estado de Madhya Pradesh, na Índia, Gajbhiye et al. (2014) realizaram análises morfométricas seguidas de técnicas de priorização. Foram considerados os seguintes parâmetros morfométricos: frequência do riacho, textura do solo, ordem do riacho, densidade de drenagem, dentre outros. Gajbhiye et al. (2014) constatou que o uso da técnica GIS aliada à verificação de campo confere maior rapidez e credibilidade quando comparado ao método convencional. No processo de priorização das bacias, são gerados mapas de priorização e os resultados indicam as sub-bacias que estão sujeitas a erosão e maiores perdas de solo, sendo indicado ações de conservação do solo. 
Francisco et al. (2008), onde associou a erodibilidade à declividade e criou um mapa de vulnerabilidade à erosão, e lkematsua et al. (2018) que aplicaram critérios biofísicos e agroambientais para priorização de microbacias hidrográficas.

A estimativa da suscetibilidade à erosão é frequentemente obtida a partir do cruzamento dos diferentes tipos de solo, análise morfológica dos perfis, química e granulométrica de amostras do solo coletadas na bacia e classes de declividade (IKEMATSUA et al., 2018); em estudos morfométricos baseados em SIG, com diferentes software de análises como, Arcgis, ArcHydro Tool, Ilwis, Basin 4, HEC-GeoRAS, a partir de dados do Modelo Digital de Elevação (MDE) (AREFIN et al., 2020); a ferramenta InVEST (Integrated Valuation of Ecosystem Services and Trade-offs) também é frequentemente utilizada em estudos que visam avaliar os serviços ecossistêmicos em escala de bacias hidrográficas, requerendo no geral poucos dados de entrada, torna-se mais simplificada, como em Hamel et al. (2017), Yang et al. (2018) e Redhead et al. (2016).

\section{Distância da malha viária}

O aumento da produção de sedimentos resultantes de mudanças no uso da terra são problemas ambientais críticos. Há um relação vastamente conhecida entre construção de estradas e o processo acelerado de erosão do solo, que exerce influência sobre a qualidade e quantidade de água superficial em reservatórios (MAHONEY et al., 2018; VETTORAZZI et al., 2016). A construção de estradas gera consideráveis impactos aos ecossistemas, devido à conversão da cobertura florestal em superfícies artificiais e impermeáveis, bem como a fragmentação espacial das paisagens urbanas com influências diretas no ambiente ecológico regional, acelerando a destruição de habitats e degradação do ecossistema (VALENTE et al., 2017).

Segundo Valente et al. (2017) e Bochner (2010) em projetos de priorização de áreas visando a conservação e preservação florestal deve-se priorizar áreas mais distantes da malha viária. Contudo, o estudo desenvolvido por Vettorazzi et al. (2016) sugere que as ações de restauração florestal sejam desenvolvidas próximo às estradas, quando localizadas junto às encostas, visando minimizar o impacto da erosão em eventos de maior pluviosidade, onde sedimentos tendem a atingir a rede de drenagem.

O reconhecimento das áreas próximas a malhas viárias é alcançado a partir do plano de informação da malha viária obtido através de imagens aéreas de satélite, análises geoespaciais de MDE, complementando o trabalho de campo (VETTORAZZI et al., 2016). São gerados mapas de distância sobre a malha viária, onde em alguns casos serão associados ao limite da bacia para obter essas distâncias somente dentro área da bacia, bem como a distância dos fragmentos florestais em relação às mesmas. Os mapas são normalizados em relação ao fator, por uma função linear crescente ou decrescente, obtendo uma relação de prioridade (VALENTE et al., 2017; BOCHNER, 2010).

\section{Índice de urbanização}

O processo de urbanização apresenta significativas alterações aos fragmentos florestais e diferentes impactos nos ciclos hidrológicos, uma vez que ocorre perda da superfície permeável, resultando na 
diminuição da infiltração de água no solo e variações das vias naturais de drenagem (BOCHNER, 2010). Há ainda interferência na estrutura e função das paisagens originais, haja vista que são transformadas em mosaicos de remanescentes florestais, produzindo desequilíbrios ecossistêmicos ao meio (VALENTE et al., 2017)

Valente et al. (2017) e Francisco (2006) sugerem que as ações de conservação e preservação florestal, assim como de manutenção da biodiversidade, sejam priorizadas em áreas distantes dos centros urbanos. Acredita-se que quanto menor o acesso de pessoas às APPs ou à fragmentos de vegetação nativa, menores serão os eventos de incêndios, corte seletivo, desmatamento e depósito de lixo nessas áreas. Na priorização de terras para proteção de recursos hídricos visando o abastecimento de água na microbacia do Rio Ware, em Massachusetts, EUA, Randhir et al. (2001) também considera a proximidade à áreas residências e industriais, um fator a ser considerado no processo de priorização de áreas.

A identificação dos centros urbanos é frequentemente obtida a partir do mapeamento do uso e ocupação do solo com a sinalização dos núcleos urbanos. Os mapas com as distâncias são gerados em ambiente (SIG), podendo utilizar um algoritmo de distância euclidiana de forma a priorizar as áreas mais afastadas dos núcleos urbanos (BOCHNER, 2010). No que tange a tomada de decisão, utiliza-se a técnica da Análise Hierárquica Analítica (AHA), onde os especialistas atribuem pesos usando escala de comparação (RANDHIR et al., 2001).

\section{Proteção de áreas ecologicamente relevantes}

As APPs são espaços especialmente protegidos atribuídos pelo Código Florestal Brasileiro (Lei 12.651/2012). Essas áreas podem ser cobertas ou não por vegetação nativa, e desempenham a "função ambiental de preservar os recursos hídricos, a paisagem, a estabilidade geológica e a biodiversidade, facilitar o fluxo gênico de fauna e flora, proteger o solo e assegurar o bem-estar das populações" (BRASIL, 2012). Garantir a preservação desses espaços protegidos no entorno dos corpos hídricos é um desafio, uma vez que a ocupação irregular, os desmatamentos e uso urbano interferem diretamente na dinâmica hidrológica, promovendo enchentes, assoreamentos e alterações no regime de vazão dos corpos hídricos.

Este critério é um importante indicador para seleção de AP, sobretudo para aquelas que apresentam aspectos de fragilidade, representatividade e potencial para recuperação de bacias hidrográficas. A recomposição ambiental em APPs propicia inúmeros benefícios aos corpos hídricos, como, a reabilitação dos ecossistemas aquáticos, controle dos processos erosivos e redução dos impactos gerados pela poluição agrícola (TURUNEN et al., 2019).

Ao selecionar sub-bacias hidrográficas dentro da bacia do ribeirão das Anhumas para recuperação ambiental em APP, Francisco et al. (2008) empregou a AMC aplicada a dois outros métodos, o da Programação por Compromisso e o da Teoria dos Jogos Cooperativos. Os dois métodos produzem uma ponderação para cada critério de acordo com o objetivo pretendido. No total, ele utilizou 13 critérios, tais quais, área da bacia, urbanização da bacia, áreas com risco de inundação, capacidade de uso, corredores de vegetação, densidade de Cursos d'Água, vazão média, matas ciliares, dentre outros. Embora haja 
objetividade nas observações, as distorções nas análises são inevitáveis, sendo imprescindível a percepção do tomador de decisão.

Para elaborar um mapa de priorização para recuperação de APPs na Bacia Hidrográfica do Córrego Jararaca, no município de São Carlos, Estado de São Paulo, Brasil, Fabbro Neto et al. (2014) utilizaram o SIG com a AMC, visando comparar esses resultados com os obtidos no mapa de diretrizes ambientais proposto no Plano de Bacia do Córrego Jararaca. Para tanto, foram aplicados 8 critérios baseados em investigação bibliográfica prévia, onde consideraram os fatores físicos e bióticos da região, a saber: proximidade de remanescente de vegetação nativa; proximidade de núcleos urbanos; uso da terra; vulnerabilidade a erosão; proximidade de malha viária; capacidade de sustentabilidade do solo; ordem de canais de drenagem e categoria de APP. Como resultado, foi observado que a escolha dos critérios para seleção de AP deve acorrer segundo os objetivos do estudo, nesse sentido, não recomenda-se selecionar critérios sem levar em consideração a ação pretendida e os interesses envolvidos no projeto.

A presença desse critério nas análises de seleção de AP garante que o percentual de restauração das APPs exigido pela legislação seja atendido, sobretudo em APPs desprovidas de cobertura florestal. Possibilita também a identificação de áreas consideradas críticas, onde medidas mitigadoras de reflorestamento devem ser incorporadas a curto prazo. Ainda não há regulamentações específicas em nível federal acerca do mecanismo de Pagamentos por Serviços Ambientais no Brasil, contudo vários estados têm adotado o PSA em leis existentes ou vinculados às normativas estabelecidas pela Lei 12.651/2012. Em projetos de PSA, a localização e percentual de APP na área são considerados critérios válidos para o processo de hierarquização (INEA, 2018; SILVA, 2018)

\section{Ponderação dos critérios}

Devido ao aprimoramento das abordagens baseadas no SIG, a sua capacidade e inúmeras tecnologias puderam ser incorporadas, o que levou à integração de sistemas GIS com modelos de análise espacial, planejamento e técnicas de tomada de decisão. As condutas mais relevantes para análise de adequação do uso da terra baseada em SIG são: mapeamento de sobreposição assistido por computador; métodos de AMC; e o métodos de inteligência artificial (MALCZEWSKI, 2004; VETTORAZZI et al., 2016).

No processo de identificação de AP, a AMC baseado em SIG destaca-se devido ao processo de combinação de dados espaciais (planos de informações) onde resultam em mapas finais que contribuem com a tomada de decisão (MALCZEWSKI, 2004; MAFRA et al., 2020). Trata-se de uma abordagem que permite a utilização de diversos critérios que devem ser avaliados a fim de alcançar um ou vários objetivos. De maneira geral, eles retratam os aspectos críticos da paisagem, os quais viabilizam a utilização e manipulação de dados geográficos, planilhas, software estatístico, incorporados ao SIG, considerando as preferências do tomador de decisão de acordo com as diretrizes estabelecidas previamente (VETTORAZZI et al., 2016).

Vários métodos de AMC foram empregados em ambiente SIG ao longo dos anos, especialmente a Combinação Linear Ponderada (CLP), a Booleana e a Média Ponderada Ordenada (MPO), as quais tem sido utilizada em múltiplas aplicações na definição de AP e suscetíveis (MALCZEWSKI, 2004; SARTORI et al., 2012; 
ALMEIDA et al., 2019). A ponderação dos critérios ocorre através da atribuição de pesos (valores) que assumem a função de diferenciar os critérios de acordo com a sua importância ou relevância na análise.

\section{CONCLUSÕES}

Os estudos sobre a identificação de APC em bacias hidrográficas encontram-se dispersos na literatura. E a pequena quantidade de artigos científicos publicados dentro dessa temática, ao longo dos últimos dez anos, retratam estudos de caráter taxonômico e ecossistêmico. As pesquisas realizadas em bacias hidrográficas possuem enfoques diversos sob múltiplos usos e não estabelecem uma relação direta entre áreas prioritárias para a conservação e restauração, evidenciando uma lacuna no conhecimento acerca de critérios objetivos para seleção dessas áreas.

A revisão de literatura demonstra que, para seleção de AP há eleição de um conjunto de critérios ou em alguns casos, de um único critério, que são avaliados e ponderados segundo objetivo da conservação. A elegibilidade dos critérios poderá ser mais efetiva mediante pesquisa bibliográfica e escolha de especialistas técnicos familiarizados com o processo de indicação de locais prioritários. Critérios abrangentes e diversificados, aliados às características ambientais, sociais e políticas da região estudada são altamente recomendáveis.

Os projetos e estudos alcançados por essa pesquisa contemplam critérios associados a áreas de captação para abastecimento público, proximidade à rede de drenagem, possibilidade de conexão de fragmentos florestais, áreas com suscetibilidade a erosão, distância da malha viária, índice de urbanização e proteção de áreas ecologicamente relevantes. Acreditamos que o emprego da abordagem multicritério incorporada ao SIG, com a integração de mapas, seja a mais adequada. É comum o uso de múltiplos critérios para atender a um ou vários objetivos simultaneamente, nas mais variadas áreas de aplicação.

Por fim, reiteramos que, os critérios levantados nesta pesquisa, podem apoiar no conhecimento acerca da identificação de áreas prioritárias, garantindo não somente a conservação e as ações de restauração, mas também o uso adequado de bacias hidrográficas, visando benefício dos recursos hídricos. Portanto, é desejável que os critérios selecionados sejam combinados com informações espaciais, instrumentos de apoio à decisão, com manipulação e análise adequada dos dados.

\section{REFERÊNCIAS}

ALMEIDA, F. C. D.; SILVEIRA, E. M. D. O.; PAIVA, L. L. D.; ACERBI JÚNIOR, F.. Mapping priority areas for forest recovery using multicriteria analysis in the brazilian atlantic forest. Raega - O Espaço Geográfico em Análise, v.46, n.3, p.113-124, 2019. DOI:

http://dx.doi.org/10.5380/raega.v46i3.67075

AREFIN, R.; MOHIR, M. I.; ALAM, J.. Watershed prioritization for soil and water conservation aspect using GIS and remote sensing: PCA-based approach at northern elevated tract Bangladesh. Applied Water Science, v.10, n.4, p.1-19, 2020. DOI: https://doi.org/10.1007/s13201-020-1176-5

BARBOSA, K. V. A. D. C.; KNOGGE, C.; DEVELEY, P. F.;
JENKINS, C. N.; UEZU, A.. Use of small Atlantic Forest fragments by birds in Southeast Brazil. Perspectives In Ecology And Conservation, v.15, n.1, p.42-46, 2017. DOI: https://doi.org/10.1016/j.pecon.2016.11.001

BEGER, M.; MCGOWAN, J.; TREML, E. A.; GREEN, A. L.; WHITE, A. T.; WOLFF, N. H.; KLEIN, C. J.; MUMBY, P. J.; POSSINGHAM, H. P.. Integrating regional conservation priorities for multiple objectives into national policy. Nature Communications, v.6, n.1, p.1-8, 2015. Dol: http://dx.doi.org/10.1038/ncomms9208

BIDEGAIN, I.; CERDA, C.; CATALÁN, E.; TIRONI, A.; LÓPEZ-

SANTIAGO, C.. Social preferences for ecosystem services in a 
biodiversity hotspot in South America. Plos One, v.14, n.4, p.1-12, 2019. DOI:

http://dx.doi.org/10.1371/journal.pone.0215715

BOCHNER, J. K.. Proposta Metodológica para Identificação de Áreas Prioritárias para Recomposição Florestal - Estudo de Caso: Bacia Hidrográfica do rio Macacu/RJ. Dissertação (Mestrado em Ciências Ambientais e Florestais) -

Universidade Rural do Rio de Janeiro, Rio de Janeiro, 2010.

BRASIL. Lei Federal n. 12.651, de 25 de maio de 2012. Dispõe sobre a proteção da vegetação nativa; e dá outras providências. Brasília: DOU, 2012.

BRASIL. Ministério do Meio Ambiente. Áreas Prioritárias para Conservação da Biodiversidade Brasileira. Brasília: MMA, 2017.

BRASIL. Ministério do Meio Ambiente. Deliberação CONABIO n. 39, de 14 de dezembro de 2005. Dispõe Sobre a Aprovação da Metodologia Para Revisão das Áreas Prioritárias Para A Conservação, Utilização Sustentável e Repartição de Benefícios da Biodiversidade Brasileira. Brasília: MMA, 2005.

BUCKLEY, L. B.; HURLBERT, A. H.; JETZ, W.. Broad-scale ecological implications of ectothermy and endothermy in changing environments. Global Ecology And Biogeography, v.21, n.9, p.873-885, 2012. DOI:

http://dx.doi.org/10.1111/i.1466-8238.2011.00737.x

CALEGARI, L.; MARTINS, S. V.; GLERIANI, J. M.; SILVA, E.; BUSATO, L. C.. Análise da dinâmica de fragmentos florestais no município de Carandaí, MG, para fins de restauração florestal. Revista Árvore, v.34, n.5, p.871-880, 2010. DOI: http://dx.doi.org/10.1590/s0100-67622010000500012

CAMACHO, A.. Respostas dos animais ectotermos terrestres à variação microclimática. Revista da Biologia, v.8, p.5-14, 2012. DOI: http://dx.doi.org/10.7594/revbio.08.02

CASSIANO, C. C.; FERRAZ, S. F. D. B.; MOLIN, P. G.; VOIGTLAENDER, M.; FERRAZ, K. M. P. M. D. B.. Spatial Assessment of Water-Related Ecosystem Services to Prioritize Restoration of Forest Patches. Natureza \& Conservação, v.11, n.2, p.176-180, 2013. DOI: http://dx.doi.org/10.4322/natcon.2013.027

COMINO, E.; BOTTERO, M.; POMARICO, S.; ROSSO, M.. The combined use of Spatial Multicriteria Evaluation and stakeholders analysis for supporting the ecological planning of a river basin.Land Use Policy, v.58, p.183-195, 2016. DOI: https://doi.org/10.1016/j.landusepol.2016.07.026

COSTA, D. D. A.; ASSUMPÇÃO, R. D. S. F. V.; AZEVEDO, J. P. S. D.; SANTOS, MARCO A. D.. Dos instrumentos de gestão de recursos hídricos - o Enquadramento - como ferramenta para reabilitação de rios. Saúde em Debate, v.43, n.3, p.3550, 2019. DOI: http://dx.doi.org/10.1590/0103$\underline{11042019 s 303}$

FRANCISCO, C. E. D. S.. Áreas de Preservação Permanente na bacia do ribeirão das Anhumas: estabelecimento de prioridades para recuperação por meio de análise multicriterial. Dissertação (Mestrado) - Instituto Agronômico, São Paulo, 2006.
FRANCISCO, C. E. D. S.; COELHO, R. M.; TORRES, R. B.; ADAMI, S. F.. Análise multicriterial na seleção de bacia hidrográfica para recuperação ambiental. Ciência Florestal, v.18, n.1, p.1-13, 2008. DOI:

http://dx.doi.org/10.5902/19805098506

FRANTZESKAKI, N.. Seven lessons for planning nature-based solutions in cities. Environmental Science \& Policy, v.93, p.101-111, 2019. DOI:

http://dx.doi.org/10.1016/i.envsci.2018.12.033

GAJBHIYE, S.; MISHRA, S. K.; PANDEY, A.. Prioritizing erosionprone area through morphometric analysis: an RS and GIS perspective. Applied Water Science, n.4, p.51-61, 2014. DOI: https://doi.org/10.1007/s13201-013-0129-7

GASHAW, T.; TULU, T.; ARGAW, M.. Erosion risk assessment for prioritization of conservation measures in Geleda watershed, Blue Nile basin, Ethiopia. Environmental Systems Research, v.6, n.1, p.1-11, 2018. DOI: https://doi.org/10.1186/s40068-016-0078-x

GJORUP, A. F.; FIDALGO, E. C. C.; PRADO, R. B.; SCHULER, A. E.. Análise de procedimentos para seleção de áreas prioritárias em programas de pagamento por serviços ambientais hídricos. Revista Ambiente e Água, v.9, n.3, p.445-458, 2014. DOI: https://doi.org/10.4136/ambiagua. 1782

HAMEL, P.; FALINSKI, K.; SHARP, R.; AUERBACH, D. A.; SÁNCHEZ-CANALES, M.; DENNEDY-FRANK, P. J.. Sediment delivery modeling in practice: Comparing the effects of watershed characteristics and data resolution across hydroclimatic regions. Science of The Total Environment, v.580, p.1381-1388, 2017.

https://doi.org/10.1016/j.scitotenv.2016.12.103

HOEK, J. V. D.; OZDOGAN, M.; BURNICKI, A.; ZHU, A..

Evaluating forest policy implementation effectiveness with a cross-scale remote sensing analysis in a priority conservation area of Southwest China. Applied Geography, v.47, p.177189, 2014. DOI:

http://dx.doi.org/10.1016/j.apgeog.2013.12.010

IGAM. Instituto Mineiro de Gestão das Águas. Gestão de bacias hidrográficas: critérios para definição de áreas prioritárias para revitalização. Belo Horizonte: IGAM, 2018.

IKEMATSUA, P.; MONTEIRO, A. C. M. C.; MACHADO, A. R.; SIQUEIRA, A. G.; TERRELLA, D.; FACCINIA, L. G.; LONGO, M. H. C.; CORREA, N. F.; ARGENTINA, P. M.; TAVARES, T. L. S.. Critérios para a priorização de microbacias hidrográficas: subsídios à implantação de esquemas de pagamentos por serviços ambientais hídricos. Revista Ipt Tecnologia e Inovação, v.2, n.7, p.59-84, 2018.

INEA. Instituto Estadual do Ambiente. Atlas dos mananciais de abastecimento público do Estado do Rio de Janeiro: subsídios ao planejamento e ordenamento territorial. Rio de Janeiro: INEA, 2018.

LIQUETE, C.; UDIAS, A.; CONTE, G.; GRIZZETTI, B.; MASI, F.. Integrated valuation of a nature-based solution for water pollution control. Highlighting hidden benefits. Ecosystem Services, v.22, p.392-401, 2016. DOI: http://dx.doi.org/10.1016/j.ecoser.2016.09.011 
LIU, J.; COOMES, D. A.; GIBSON, L.; HU, G.; LIU, J.; LUO, Y; WU, C.; YU, M.. Forest fragmentation in China and its effect on biodiversity. Biological Reviews, v.94, n.5, p.1636-1657, 2019. DOI: http://dx.doi.org/10.1111/brv.12519

MAFRA, R.; OSCO, L. P.; ALVES, M. R.; RAMOS, A. P.. Definição de áreas prioritárias para a recuperação florestal em Bacias Hidrográficas a partir de análise multicritério. Caminhos de Geografia, Uberlândia, v.21, n.77, p.220-233, 2020. DOI: https://doi.org/10.14393/RCG217752869

MAHONEY, D. T.; FOX, J. F.; AAMERY, N.. Watershed erosion modeling using the probability of sediment connectivity in a gently rolling system. Journal of Hydrology, v.561, p.862883, 2018. DOI:

https://doi.org/10.1016/j.jhydrol.2018.04.034

MALCZEWSKI, J.. GIS-based multicriteria decision analysis: a survey of the literature. International Journal of Geographical Information Science, v.20, n.7, p.703-726, 2006. DOI: http://dx.doi.org/10.1080/13658810600661508

MARGULES, C. R.; PRESSEY, R. L.. Systematic conservation planning. Nature, Camberra, v.405, n.6783, p.243-253, 2000. DOI: http://dx.doi.org/10.1038/35012251

MATSUOKA, E. H.. Conservação de água através de pagamento por serviços ambientais: avaliação de fatores críticos de sucesso dos projetos do rio camboriú e das cidades de extrema e Nova lorque. Dissertação (Mestrado profissional MPGC) - Fundação Getulio Vargas Escola de Administração de Empresas de São Paulo Edson, 2019.

MELLO, K. D.; COSTA, D. R. D. A.; VALENTE, R. A.; VETTORAZZI, C. A.. Multicriteria Evaluation for Protected Area Definition Aiming at Water Quality Improvement. Floresta e Ambiente, São Paulo, v.25, n.3, p.1-10, 2018. DOI: https://doi.org/10.1590/2179$\underline{8087.013416}$

MESHRAM, S. G.; ALVANDI, E.; SINGH, V. P.; MESHRAM, C.. Comparison of AHP and fuzzy AHP models for prioritization of watersheds. Soft Computing, v.23, n.24, p.13615-13625, 2019. DOI: http://dx.doi.org/10.1007/s00500-019-03900-z

MESHRAM, S. G.; SHARMA, S. K.. Prioritization of watershed through morphometric parameters: a pca-based approach. Applied Water Science, v.7, n.3, p.1505-1519, 2017. DOI: http://dx.doi.org/10.1007/s13201-015-0332-9

METZGER, J. P.; CASATTI, L.. Do diagnóstico à conservação da biodiversidade: o estado da arte do programa BIOTA/FAPESP. Biota Neotropica, São Paulo, v.6, n.2, p.1-23, 2006.

MITCHELL, M. G. E.; SUAREZ-CASTRO, A. F.; MARTINEZHARMS, M.; MARON, M.; MCALPINE, C.; GASTON, K. J.; JOHANSEN, K.; RHODES, J. R.. Reframing landscape fragmentation's effects on ecosystem services. Trends In Ecology \& Evolution, v.30, n.4, p.190-198, 2015. DOI: https://doi.org/10.1016/j.tree.2015.01.011

MOKANY, K.; WESTCOTT, D. A.; PRASAD, S.; FORD, A. J.; METCALFE, D. J.. Identifying Priority Areas for Conservation and Management in Diverse Tropical Forests. Plos One, v.9, n.2, p.1-10, 2014. DOI:

http://dx.doi.org/10.1371/journal.pone.0089084
MORI, G. B.; PAULA, F. R.; FERRAZ, S. F. B.; CAMARGO, A. F. M.; MARTINELLI, L. A.. Influence of landscape properties on stream water quality in agricultural catchments in Southeastern Brazil. Annales de Limnologie: International Journal of Limnology, v.51, n.1, p.11-21, 2015. DOI: https://doi.org/10.1590/S2179-975X2011000200009

MYERS, N.. The biodiversity challenge: Expanded hot-spots analysis. The Environmentalist, v.10, n.4, p.243-256, 1990. DOI: http://dx.doi.org/10.1007/bf02239720

MYERS, N.. Threatened biotas. The Environmentalist, v.8, n.3, p.187-208, 1988. DOI: http://dx.doi.org/10.1007/bf02240252

MYERS, N.; MITTERMEIER, R. A.; MITTERMEIER, C. G.; FONSECA, G. A. B. D.; KENT, J.. Biodiversity hotspots for conservation priorities. Nature, v.403, n.6772, p.853-858, 2000. DOI: http://dx.doi.org/10.1038/35002501

FABBRO NETO, F.; MARQUES, E. M.; SANTOS, F. S.; MONTAÑO, M.. Critérios técnicos e de participação social para a recuperação florestal: Quais as diferenças na definição de áreas prioritárias?. Engenharia Sanitária e Ambiental, v.19, n.4, p.353-360, 2014. DOI: http://dx.doi.org/10.1590/S1413-41522014019000000403

NÉTO, N. C. G.; SOUZA, L. D. N. D.; CASTRO, C. A. F.; COSTA, D. D. A.; FERREIRA, M. I. P.. Soluções Baseadas na Natureza aplicadas à conservação e à gestão integrada das águas: um estudo prospectivo à luz da Agenda 2030 da ONU. Revista Principia, v.1, n.51, p.30-42, 2020. DOI: http://dx.doi.org/10.18265/1517-0306a2020v1n51p30-43

PAGIOLA, S.; VON GLEHN, H. C.; TAFFARELLO,

D.. Experiências de pagamentos por serviços ambientais no Brasil. São Paulo: Secretaria do Meio Ambiente de São Paulo, 2013.

PARDINI, R.; BUENO, A. D. A.; GARDNER, T. A.; PRADO, P. I .; METZGER, J. P.. Beyond the Fragmentation Threshold Hypothesis: regime shifts in biodiversity across fragmented landscapes. Plos One, v.5, n.10, p.1-10, 2010. DOI: https://doi.org/10.1371/journal.pone.0013666

PAUDYAL, K.; BARAL, H.; BHANDARI, S. P.; BHANDARI, A.; KEENAN, R. J.. Spatial assessment of the impact of land use and land cover change on supply of ecosystem services in Phewa watershed, Nepal. Ecosystem Services, v.36, p.100895, 2019. DOI:

https://doi.org/10.1016/i.ecoser.2019.100895

PINHEIRO, J. A. C.; DIAS, H. C. T.; PAIVA, H. N.; SILVA, C. M.; FERNANDES FILHO, E. I.; OLIVEIRA NETO, S. N.; BARBOSA, R. A.. Processos hidrológicos na bacia hidrográfica do Córrego Zerede em Timóteo-MG. Ciência Florestal, v.29, n.4, p.16581670, 2019. DOI: http://dx.doi.org/10.5902/198050984677

POSTEL, S. L.; THOMPSON, B. H.. Watershed protection: capturing the benefits of nature's water supply services. Natural Resources Forum, v.29, n.2, p.98-108, 2005. DOI: https://doi.org/10.1111/j.14778947.2005.00119.x

QIU, J.; TURNER, M. G.. Importance of landscape heterogeneity in sustaining hydrologic ecosystem services in 
an agricultural watershed. Ecosphere, v.6, n.11, p.1-19, 2015. DOI: http://dx.doi.org/10.1890/ES15-00312.1

RANDHIR, T. O.; O'CONNOR, R.; PENNER, P. R.; GOODWIN, D. W.. A watershed-based land prioritization model for water supply protection.Forest Ecology and Management, v.143, n.1-3, p.47-56, 2001. DOI: http://dx.doi.org/10.1016/s0378-1127(00)00504-1

RAPACCIUOLO, G.. Strengthening the contribution of macroecological models to conservation practice. Global Ecology and Biogeography, v.28, n.1, p.54-60, 2018. DOI: https://doi.org/10.1111/geb.12848

REDHEAD, J. W.; STRATFORD, C.; SHARPS, K.; JONES, L.; ZIV, G.; CLARKE, D.; OLIVER, T. H.; BULLOCK, J. M.. Empirical validation of the InVEST water yield ecosystem service model at a national scale. Science Of The Total Environment, v.569-570, p.1418-1426, 2016. DOI: http://dx.doi.org/10.1016/j.scitotenv.2016.06.227

REID, W. V.. Biodiversity hotspots. Trends In Ecology \& Evolution, v.13, n.7, p.275-280, 1998. DOI: http://dx.doi.org/10.1016/s0169-5347(98)01363-9

RIO DE JANEIRO. Decreto n. 42.029, de 15 de junho de 2011. Regulamenta o Programa Estadual de Conservação revitalização de recursos hídricos - PROHIDRO, previsto nos artigos 50 e 11 da lei no 3.239, de 02 de agosto de 1999, que instituiu a Política Estadual de recursos hídricos, e dá outras providências. Rio de Janeiro: DOE, 2011.

RIO DE JANEIRO. Lei n. 3.239, de 02 de agosto de 1999. Institui a Política Estadual de Recursos Hídricos; cria o Sistema Estadual de gerenciamento de recursos hídricos; regulamenta a Constituição Estadual, em seu artigo 261, parágrafo 10 , inciso vii; e dá outras providências. Rio de Janeiro: DOE, 1999.

ROUX, J. J. L. E.; HUI, C.; CASTILLO, M. L.; IRIONDO, J. M.; KEET, J. H.; KHAPUGIN, A. A.; MÉDAIL, F.; REJMÁNEK, M.; THERON, G.; YANNELLI, F. A.. Recent anthropogenic plant extinctions differ in biodiversity hotspots and coldspots. Current Biology, v.29, n.17, p.2912-2918, 2019. DOI: http://dx.doi.org/10.1016/j.cub.2019.07.063

RUIZ, C. B.. Pagamento por serviços ambientais: da teoria à prática. Rio Claro: ITPA, 2015.

SANTOS, F. A. M. D.; COELHO JUNIOR, M. G.; CARDOSO, J. C.; BASSO, V. M.; MARQUES, A. L. D. P.; SILVA, E. M. R.. Program outcomes of payments for watershed services in brazilian atlantic forest: how to evaluate to improve decision-making and the socio-environmental benefits. Water, v.12, n.9, p.2441-2452, 2020. DOI: https://doi.org/10.3390/w12092441

SARTORI, A. A. C.; SILVA, R. F. B.; ZIMBACK, C. R. L.. Combinação linear ponderada na definição de áreas prioritárias à conectividade entre fragmentos florestais em ambiente SIG. Revista Árvore, v.36, n.6, p.1079-1090, 2012. DOI: http://dx.doi.org/10.1590/s0100-67622012000600009
SILVA, A. A. D.. Panorama da aplicação de práticas de conservação de solo e água nos Programas de Pagamento por Serviços Ambientais hídricos do Estado do Rio de Janeiro. Dissertação (Mestrado em Rede Nacional em Gestão e Regulação de Recursos Hídricos) - Universidade do Estado do Rio de Janeiro, Rio de Janeiro, 2018.

SILVA, V. A. M.; MELLO, K. D.; VETTORAZZI, C. A.; COSTA, D. R.; VALENTE, R. A.. Priority areas for forest conservation, aiming at the maintenance of water resources, through the multicriteria evaluation. Revista Árvore, v.41, n.1, p.1-11, 2017. DOI: http://dx.doi.org/10.1590/1806$\underline{90882017000100019}$

SIMONCIC, T.; BONCINA, A.; ROSSET, C.; BINDER, F.; MEO, I.; CAVLOVIC, J.; GAL, J.; MATIJASIC, D.; SCHNEIDER, J.; SINGER, F.. Importance of priority areas for multi-objective forest planning: a central european perspective. International Forestry Review, v.15, n.4, p.509-523, 2013. DOI: http://dx.doi.org/10.1505/146554813809025685

TAMBOSI, L. R.; VIDAL, M. M.; FERRAZ, S. F. B.; METZGER, J. P.. Funções eco-hidrológicas das florestas nativas e o Código Florestal. Estudos Avançados, v.29, n.84, p.151-162, 2015. DOI: http://dx.doi.org/10.1590/s0103-40142015000200010

TURUNEN, J.; MARKKULA, J.; RAJAKALLIO, M.; AROVIITA, J.. Riparian forests mitigate harmful ecological effects of agricultural diffuse pollution in medium-sized streams. Science Of The Total Environment, v.649, p.495-503, 2019. DOI: https://doi.org/10.1016/j.scitotenv.2018.08.427

VALENTE, R. A.; PETEAN, F. C. S.; VETTORAZZI, C. A.. Multicriteria decision analysis for prioritizing areas for forest restoration.Cerne, v.23, n.1, p.53-60, 2017. DOI: https://doi.org/10.1590/01047760201723012258

VETTORAZZI, C. A.; VALENTE, R. A.. Priority areas for forest restoration aiming at the conservation of water resources. Ecological Engineering, São Paulo, v.94, p.255267, 2016. DOI:

http://dx.doi.org/10.1016/i.ecoleng.2016.05.069

WILLIAMS, K. J.; MARGULES, C. R.; PERT, P. L.; BARRETT, T.. A preliminary Assessment of Priority Areas for Plant Biodiversity Conservation in the Wet Tropics Bioregion. Living in a Dynamic Tropical Forest Landscape, p.577-590, 2009. DOI: http://dx.doi.org/10.1002/9781444300321.ch46

WOOD, S. L. R.; JONES, S. K.; JOHNSON, J. A.; BRAUMAN, K. A.; CHAPLIN-KRAMER, R.; FREMIER, A.; GIRVETZ, E.; GORDON, L. J.; KAPPEL, C. V.; MANDLE, L.. Distilling the role of ecosystem services in the Sustainable Development Goals. Ecosystem Services, v.29, p.70-82, 2018. DOI: http://dx.doi.org/10.1016/i.ecoser.2017.10.010

YANG, S.; ZHAO, W.; LIU, Y.; WANG, S.; WANG, J.; ZHAI, R. Influence of land use change on the ecosystem service tradeoffs in the ecological restoration area: Dynamics and scenarios in the Yanhe watershed, China. Science of The Total Environment, v.644, p.556-566, 2018. DOI: https://doi.org/10.1016/j.scitotenv.2018.06.348

A CBPC - Companhia Brasileira de Produção Científica (CNPJ: 11.221.422/0001-03) detém os direitos materiais desta publicação. Os direitos referem-se à publicação do trabalho em qualquer parte do mundo, incluindo os direitos às renovações, expansões e disseminações da contribuição, bem como outros direitos subsidiários. Todos os trabalhos publicados eletronicamente poderão posteriormente ser publicados em coletâneas impressas sob coordenação da Sustenere Publishing, da Companhia Brasileira de Produção Científica e seus parceiros autorizados. Os (as) autores (as) preservam os direitos autorais, mas não têm permissão para a publicação da contribuição em outro meio, impresso ou digital, em português ou em tradução. 